\title{
DAUGAVET- AND DELTA-POINTS IN BANACH SPACES WITH UNCONDITIONAL BASES
}

\author{
TROND A. ABRAHAMSEN, VEGARD LIMA, ANDRÉ MARTINY, \\ AND STANIMIR TROYANSKI
}

\begin{abstract}
We study the existence of Daugavet- and delta-points in the unit sphere of Banach spaces with a 1-unconditional basis. A norm one element $x$ in a Banach space is a Daugavet-point (resp. delta-point) if every element in the unit ball (resp. $x$ itself) is in the closed convex hull of unit ball elements that are almost at distance 2 from $x$. A Banach space has the Daugavet property (resp. diametral local diameter two property) if and only if every norm one element is a Daugavet-point (resp. delta-point). It is well-known that a Banach space with the Daugavet property does not have an unconditional basis. Similarly spaces with the diametral local diameter two property do not have an unconditional basis with suppression unconditional constant strictly less than 2 .

We show that no Banach space with a subsymmetric basis can have deltapoints. In contrast we construct a Banach space with a 1-unconditional basis with delta-points, but with no Daugavet-points, and a Banach space with a 1-unconditional basis with a unit ball in which the Daugavet-points are weakly dense.
\end{abstract}

\section{INTRODUCTION}

Let $X$ be a Banach space with unit ball $B_{X}$, unit sphere $S_{X}$, and topological dual $X^{*}$. For $x \in S_{X}$ and $\varepsilon>0$ let $\Delta_{\varepsilon}(x)=\left\{y \in B_{X}:\|x-y\| \geq 2-\varepsilon\right\}$. We say that $X$ has the

(i) Daugavet property if for every $x \in S_{X}$ and every $\varepsilon>0$ we have $B_{X}=$ $\overline{\operatorname{conv}} \Delta_{\varepsilon}(x)$

(ii) diametral local diameter two property if for every $x \in S_{X}$ and every $\varepsilon>0$ we have $x \in \overline{\operatorname{conv}} \Delta_{\varepsilon}(x)$.

In Kad96, Corollary 2.3] Kadets proved that any Banach space with the Daugavet property fails to have an unconditional basis (see also Wer01, Proposition 3.1]). These arguments are probably the easiest known proofs of the absence of unconditional bases in the classical Banach spaces $C[0,1]$ and $L_{1}[0,1]$. The diametral local diameter two property was named and studied in BGLPRZ18, but it was first introduced in IK04 under the name space with bad projections. (See the references in IK04 for previous unnamed appearances of this property.) Using the characterizations in [K04] we see that if a Banach space with the diametral local

Received by the editors July 11, 2020, and, in revised form, January 18, 2021.

2020 Mathematics Subject Classification. Primary 46B20, 46B22, 46 B04.

Key words and phrases. Delta-point, Daugavet-point, diametral diameter two property, Daugavet property, 1-unconditional basis.

The fourth-named author was supported by MTM2017-86182-P (AEI/FEDER, UE), and Bulgarian National Scientific Fund, Grant, KP-06-H22/4, 04.12.2018. 
diameter two property has an unconditional basis, then the unconditional suppression basis constant is at least 2. But note that we do not know of any Banach space with an unconditional basis and the diametral local diameter two property.

In the present paper we study pointwise versions of the Daugavet property and the diametral local diameter two property in spaces with 1-unconditional bases.

Definition 1.1. Let $X$ be a Banach space and let $x \in S_{X}$. We say that $x$ is

(i) a Daugavet-point if for every $\varepsilon>0$ we have $B_{X}=\overline{\operatorname{conv}} \Delta_{\varepsilon}(x)$;

(ii) a delta-point if for every $\varepsilon>0$ we have $x \in \overline{\operatorname{conv}} \Delta_{\varepsilon}(x)$.

Daugavet-points and delta-points were introduced in AHLP20. For the spaces $L_{1}(\mu)$, for preduals of such spaces, and for Müntz spaces these notions are the same AHLP20, Theorems 3.1, 3.7, and 3.13]. However, $C[0,1] \oplus_{2} C[0,1]$ is an example of a space with the diametral local diameter two property, but with no Daugavetpoints AHLP20, Example 4.7]. Stability results for Daugavet- and delta-points in absolute sums of Banach spaces was further studied in [HPV20].

In Section 2 we consider Banach spaces with 1-unconditional bases and study a family of subsets of the support of a vector $x$. We find properties of these subsets that are intimately linked to $x$ not being a delta-point. Quite general results are obtained in this direction. We apply these results to show that Banach spaces with subsymmetric bases (these include separable Lorentz and Orlicz sequence spaces) always fail to contain delta-points.

In Section 3 we construct a Banach space with a 1-unconditional basis which contains a delta-point, but contain no Daugavet-points. The example is a Banach space of the type $h_{\mathcal{A}, 1}$ generated by an adequate family of subsets of a binary tree. The norm of the space is the supremum of the $\ell_{1}$-sum of branches in the binary tree.

In Section 4 we modify slightly the binary tree from Section 3 and the associated adequate family, to obtain an $h_{\mathcal{A}, 1}$ space with some remarkable properties: It has Daugavet-points; the Daugavet-points are even weakly dense in the unit ball; the diameter of every slice of the unit ball is two, but is has relatively weakly open subsets of the unit ball of arbitrary small diameter.

Finally, let us also remark that the examples in both Section 3 and Section 4 contain isometric copies of $c_{0}$ and $\ell_{1}$. Both the $\ell_{1}$-ness of the branches and $c_{0^{-}}$ ness of antichains in the binary tree play an important role in our construction of Daugavet- and delta-points in these spaces (see e.g. Theorems 3.1 and 4.2, and Corollary 4.3).

\section{1-UNCONDITIONAL BASES AND THE SETS $M(x)$}

The main goal of this section is to prove that Banach spaces with a subsymmetric basis fail to have delta-points. Before we start this mission, let us point out some results and concepts that we will need. First some characterizations of Daugavetand delta-points that we will frequently use throughout the paper.

Recall that a slice of the unit ball $B_{X}$ of a Banach space $X$ is a subset of the form

$$
S\left(x^{*}, \varepsilon\right)=\left\{x \in B_{X}: x^{*}(x)>\left\|x^{*}\right\|-\varepsilon\right\},
$$

where $x^{*} \in X^{*}$ and $\varepsilon>0$. 
Proposition 2.1 ([AHLP20, Lemma 2.3]). Let $X$ be a Banach space and $x \in S_{X}$. The following assertions are equivalent:

(i) $x$ is a Daugavet-point;

(ii) for every slice $S$ of $B_{X}$ and for every $\varepsilon>0$ there exists $y \in S$ such that $\|x-y\| \geq 2-\varepsilon$.

Proposition 2.2 ([AHLP20, Lemma 2.2]). Let $X$ be a Banach space and $x \in S_{X}$. The following assertions are equivalent:

(i) $x$ is a delta-point;

(ii) for every slice $S$ of $B_{X}$ with $x \in S$ and for every $\varepsilon>0$ there exists $y \in S$ such that $\|x-y\| \geq 2-\varepsilon$.

Let $X$ be a Banach space. Recall that a Schauder basis $\left(e_{i}\right)_{i \in \mathbb{N}}$ of $X$ is called unconditional if for every $x \in X$ its expansion $x=\sum_{i \in \mathbb{N}} x_{i} e_{i}$ converges unconditionally. If, moreover, $\left\|\sum_{i \in \mathbb{N}} \theta_{i} x_{i} e_{i}\right\|=\left\|\sum_{i \in \mathbb{N}} x_{i} e_{i}\right\|$ for any $x=\sum_{i \in \mathbb{N}} x_{i} e_{i} \in$ $X$ and any sequence of signs $\left(\theta_{i}\right)_{i \in \mathbb{N}}$, then $\left(e_{i}\right)_{i \in \mathbb{N}}$ is called 1-unconditional. A Schauder basis is called subsymmetric, or 1-subsymmetric, if it is unconditional and $\left\|\sum_{i \in \mathbb{N}} \theta_{i} x_{i} e_{k_{i}}\right\|=\left\|\sum_{i \in \mathbb{N}} x_{i} e_{i}\right\|$ for any $x=\sum_{i \in \mathbb{N}} x_{i} e_{i} \in X$, any sequence of signs $\left(\theta_{i}\right)_{i \in \mathbb{N}}$, and any infinite increasing sequence of naturals $\left(k_{i}\right)_{i \in \mathbb{N}}$. Trivially a subsymmetric basis is 1-unconditional. In the following we will assume that the basis $\left(e_{i}\right)_{i \in \mathbb{N}}$ is normalized, i.e. $\left\|e_{i}\right\|=1$ for all $i \in \mathbb{N}$. With $\left(e_{i}^{*}\right)_{i \in \mathbb{N}}$ we denote the conjugate in $X^{*}$ to the basis $\left(e_{i}\right)_{i \in \mathbb{N}}$. Clearly $\left(e_{i}^{*}\right)_{i \in \mathbb{N}}$ is a 1-unconditional basic sequence whenever $\left(e_{i}\right)_{i \in \mathbb{N}}$ is. When studying Daugavet-points or delta-points in a Banach space $X$ with 1-unconditional basis $\left(e_{i}\right)_{i \in \mathbb{N}}$ we can restrict our investigation to the positive cone $K_{X}$ generated by the basis, where

$$
K_{X}=\left\{x=\sum_{i \in \mathbb{N}} x_{i} e_{i}: x_{i} \geq 0\right\}=\left\{x \in X: e_{i}^{*}(x) \geq 0\right\} .
$$

The reason for this is that for every sequence of signs $\theta=\left(\theta_{i}\right)_{i \in \mathbb{N}}$ the operator $T_{\theta}: X \rightarrow X$ defined by $T_{\theta}\left(\sum_{i \in \mathbb{N}} x_{i} e_{i}\right)=\sum_{i \in \mathbb{N}} \theta_{i} x_{i} e_{i}$ is a linear isometry. Hence $x=$ $\sum_{i \in \mathbb{N}} x_{i} e_{i}$ is a Daugavet-point (resp. delta-point) if and only if $|x|=\sum_{i \in \mathbb{N}}\left|x_{i}\right| e_{i}$ is.

The following result is well-known.

Proposition 2.3. Let $X$ be a Banach space with a 1-unconditional basis $\left(e_{i}\right)_{i \in \mathbb{N}}$. If $\sum_{i \in \mathbb{N}} b_{i} e_{i}$ is convergent and $\left|a_{i}\right| \leq\left|b_{i}\right|$ for all $i$, then $\sum_{i \in \mathbb{N}} a_{i} e_{i}$ is convergent and

$$
\left\|\sum_{i \in \mathbb{N}} a_{i} e_{i}\right\| \leq\left\|\sum_{i \in \mathbb{N}} b_{i} e_{i}\right\|
$$

Moreover $\left\|P_{A}\right\|=1$ where, for $A \subset \mathbb{N}, P_{A}$ is the projection defined by

$$
P_{A}\left(\sum_{i \in \mathbb{N}} x_{i} e_{i}\right)=\sum_{i \in A} x_{i} e_{i} .
$$

From this we immediately get a fact that will be applied several times throughout the paper.

Fact 2.4. Let $X$ be a Banach space with a 1-unconditional basis $\left(e_{i}\right)_{i \in \mathbb{N}}$ and let $x, y \in X$ and $E \subset \mathbb{N}$. Then the following holds.

- If $\left|x_{i}\right| \leq\left|y_{i}\right|$ and $\operatorname{sgn} x_{i}=\operatorname{sgn} y_{i}$ for all $i \in E$, then $\left\|y-P_{E} x\right\| \leq\|y\|$. 
The upshot of Fact 2.4 is that it can be used to find an upper bound for the distance between $x \in S_{X}$ and elements in a given subset of the unit ball. Indeed, suppose we can find $E \subseteq \mathbb{N}, \eta>0$ and a subset $S$ of the unit ball such that $\left\|x-P_{E} x\right\|<1-\eta$ and the assumption in Fact 2.4 holds for any $y \in S$. Then

$$
\|x-y\| \leq\left\|x-P_{E} x\right\|+\left\|y-P_{E} x\right\|<2-\eta \text {. }
$$

If such a set $S$ is a slice (resp. a slice containing $x$ ), then $x$ cannot be a Daugavetpoint (resp. delta-point). We will see in Theorem 2.17 that any unit sphere element in a space with a subsymmetric basis, is contained in a slice of the above type. Our tool to investigate the existence of slices of this type in a Banach space with a 1-unconditional basis, are certain families of subsets of the support of the elements in the space.

Remark 2.5. If only the moreover part of Proposition 2.3 holds, then the basis is called 1-suppression unconditional. In this case the conclusion of Proposition 2.3 still holds if $\operatorname{sgn} a_{i}=\operatorname{sgn} b_{i}$, for all $i$. This is all that is needed in Fact 2.4. Similarly, one can check that all the results about 1-unconditional bases in the rest of this section also holds for a Banach space $X$ with a 1-suppression unconditional basis.

Definition 2.6. For any Banach space $X$ with 1-unconditional basis $\left(e_{i}\right)_{i \in \mathbb{N}}$ and for $x \in X$, define

$$
\begin{gathered}
M(x):=\left\{A \subseteq \mathbb{N}:\left\|P_{A} x\right\|=\|x\|,\left\|P_{A} x-x_{i} e_{i}\right\|<\|x\|, \text { for all } i \in A\right\}, \\
M^{\mathcal{F}}(x):=\{A \in M(x):|A|<\infty\},
\end{gathered}
$$

and

$$
M^{\infty}(x):=\{A \in M(x):|A|=\infty\} .
$$

We can think of $M(x)$ as a collection of minimal "norm-giving" subsets of the support of $x$. If for example $X=c_{0}$ and $x \in X$, then $M(x)=\left\{\{i\}:\left|x_{i}\right|=\|x\|\right\}$ while if $X=\ell_{p}, 1 \leq p<\infty$ and $x \in X$, then $M(x)=\{\operatorname{supp}(x)\}$.

Our first observation about the families $M(x)$ is that they are always non-empty.

Lemma 2.7. Let $X$ be a Banach space with 1-unconditional basis $\left(e_{i}\right)_{i \in \mathbb{N}}$. Then $M(x) \neq \emptyset$ for all $x \in X$.

Proof. Let $x \in X$. Either $A_{0}:=\operatorname{supp}(x) \in M(x)$ or there exists a smallest $n_{1} \in A_{0}$ such that if we define $A_{1}=A_{0} \backslash\left\{n_{1}\right\}$, then $\left\|P_{A_{1}} x\right\|=\|x\|$ and

$$
\left\|P_{A_{1}} x-x_{j} e_{j}\right\|<\|x\| \text { for all } j \in A_{0} \cap\left\{1, \ldots, n_{1}-1\right\} .
$$

Suppose we have found $n_{1}<\cdots<n_{k-1}$ such that $A_{k-1}=A_{k-2} \backslash\left\{n_{k-1}\right\}$ satisfies $\left\|P_{A_{k-1}} x\right\|=\|x\|$ and $\left\|P_{A_{k-1}} x-x_{j} e_{j}\right\|<\|x\|$ for all $j \in A_{k-1} \cap\left\{1, \ldots, n_{k-1}-1\right\}$. Then either $A_{k-1} \in M(x)$ or there exists a smallest integer $n_{k}$ greater than $n_{k-1}$ such that $A_{k}=A_{k-1}(x) \backslash\left\{n_{k}\right\}$ satisfies $\left\|P_{A_{k}} x\right\|=\|x\|$ and

$$
\left\|P_{A_{k}} x-x_{j} e_{j}\right\|<\|x\| \text { for all } j \in A_{k} \cap\left\{1, \ldots, n_{k}-1\right\} .
$$

Either this process terminates and $A_{k} \in M(x)$, or we get a set $N=\left\{n_{i}\right\}_{i=1}^{\infty}$. Let $A=\bigcap_{k} A_{k}=\operatorname{supp}(x) \backslash N$ and note that $\left\|P_{A} x\right\|=\|x\|$. If $j \in A$, find $k$ such that $j<n_{k}$, then by 1-unconditionality

$$
\left\|P_{A} x-x_{j} e_{j}\right\| \leq\left\|P_{A_{k}} x-x_{j} e_{j}\right\|<\|x\|
$$

and $A \in M(x)$. 
Our next goal is to prove that certain classes of subsets of $M^{\mathcal{F}}(x)$ and $M^{\infty}(x)$ are finite (see Lemma 2.10 below). We will use the next result as a stepping stone. In the proof, and throughout the paper, we will assume that the sets $A=$ $\left\{a_{1}, a_{2}, \ldots\right\} \in M(x)$ are ordered so that $a_{1}<a_{2}<\cdots<a_{n}<\cdots$, and we will use $A(n)$ to denote the set $\left\{a_{1}, \ldots, a_{n}\right\}$.

Lemma 2.8. Let $X$ be a Banach space with 1 -unconditional basis $\left(e_{i}\right)_{i \in \mathbb{N}}$. If $x \in X$, then for every $n \in \mathbb{N}$,

(i) $|\{A(n): A \in M(x),|A|>n\}|<\infty$;

(ii) $|\{A \in M(x):|A| \leq n\}|<\infty$.

In particular, $\left|\bigcup_{D \in M^{\infty}(x)}\{D(n)\}\right|<\infty$.

Proof. Let us prove (i) inductively. For $k \in \mathbb{N}$, let $R_{k}=I-P_{N_{k}}$, where $N_{k}=$ $\{1, \ldots, k\}$. For $n=1$ the result follows from $\left\|R_{k} x\right\| \rightarrow 0$.

Now assume that $|\{A(n-1): A \in M(x),|A|>n-1\}|<\infty$, and let $s_{n-1}:=$ $\max \left\{\left\|P_{A(n-1)} x\right\|: A \in M(x),|A|>n-1\right\}<\|x\|$. Find $k \in \mathbb{N}$ such that $\left\|R_{k} x\right\|<$ $\|x\|-s_{n-1}$. Then by the triangle inequality, it follows that $\max A(n) \leq k$ for all $A \in M(x)$ with $|A|>n$.

For (ii), let $A \in M(x)$ with $|A|=n$. Then $\left\|P_{A(n-1)} x\right\| \leq s_{n-1}$, and thus $\max A \leq k$, where as above $k \in \mathbb{N}$ is such that $\left\|R_{k} x\right\|<\|x\|-s_{n-1}$.

In order to find the sets $E \subseteq \mathbb{N}$ mentioned in the remarks following Fact 2.4 we need the following families of subsets of $M(x)$.

Definition 2.9. Let $X$ have 1-unconditional basis $\left(e_{i}\right)_{i \in \mathbb{N}}$. Let $x \in S_{X}$ and define

$$
\begin{aligned}
& \mathcal{F}_{n}(x):=\left\{A \in M^{\mathcal{F}}(x): A \cap D(n) \neq D(n), \text { for all } D \in M^{\infty}(x)\right\}, \\
& \mathcal{G}_{n}(x):=\mathcal{F}_{n}(x) \cup \bigcup_{D \in M^{\infty}(x)}\{D(n)\}, \\
& \mathcal{E}_{n}(x):=\left\{E \subset \bigcup_{A \in \mathcal{G}_{n}} A: E \cap A \neq \emptyset, \text { for all } A \in \mathcal{G}_{n}\right\} .
\end{aligned}
$$

If it is clear from the context what element $x$ we are considering, we will simply denote these sets by $\mathcal{F}_{n}, \mathcal{G}_{n}$, and $\mathcal{E}_{n}$.

It is pertinent with a couple of comments about these families of sets. Trivially, if $M^{\infty}(x)=\emptyset$, then $\mathcal{G}_{n}=\mathcal{F}_{n}=M(x)$ for all $n \in \mathbb{N}$. We can think of the elements of $\mathcal{E}_{n}$ as essential for the norm of $x$, i.e. $\left\|x-P_{E} x\right\|<\|x\|$ for all $E \in \mathcal{E}_{n}$. According to Lemma 2.11 below the drop in norm is also uniformly bounded away from 0 . The main reason for this is that $\mathcal{F}_{n}$ and $\mathcal{E}_{n}$ are finite for all $n \in \mathbb{N}$. We will prove this now.

Lemma 2.10. Let $X$ have 1-unconditional basis $\left(e_{i}\right)_{i \in \mathbb{N}}$. If $x \in S_{X}$, then for all $n \in \mathbb{N}$,

(i) $\left|\mathcal{F}_{n}\right|<\infty$;

(ii) $\left|\mathcal{E}_{n}\right|<\infty$.

In particular, if $M^{\infty}(x)=\emptyset$, then $|M(x)|<\infty$.

Proof. (i). There exists $N \in \mathbb{N}$ such that $\max _{D \in M^{\infty}(x)} D(n) \leq N$ by Lemma 2.8.

Assume for contradiction that $\left|\mathcal{F}_{n}\right|=\infty$. Then there exists a sequence $\left(A_{k}\right) \subset$ $\mathcal{F}_{n}$ such that $\left|A_{k}\right| \geq k$. By compactness of $\{0,1\}^{\mathbb{N}}$ and passing to a subsequence 
if necessary, we may assume that $A_{k} \rightarrow A \in \mathbb{N}$ pointwise and $A \cap\{1, \ldots, N\}=$ $A_{k} \cap\{1, \ldots, N\}$ for all $k$. In particular $\left\|P_{A} x\right\|=1$. By Lemma 2.7, there exists $B \subseteq A$, such that $B \in M\left(P_{A} x\right) \subseteq M(x)$. Since $A \cap\{1, \ldots, N\}=A_{k} \cap\{1, \ldots, N\}$, we have $|B|<\infty$ by definition of $\mathcal{F}_{n}$. Since $B$ is finite $A_{k} \cap B$ is eventually constant. Thus for some $k \in \mathbb{N}$ we have $B \subsetneq A_{k} \in M(x)$, a contradiction.

Finally, (ii) follows from (i) and Lemma 2.8 .

With the knowledge that the cardinality of $\mathcal{E}_{n}$ is finite for every $n \in \mathbb{N}$, we now obtain the following result.

Lemma 2.11. Let $X$ be a Banach space with 1-unconditional basis $\left(e_{i}\right)_{i \in \mathbb{N}}$. If $x \in S_{X}$, then

(i) $\left\|x-P_{E} x\right\|<1$ if $E \cap A \neq \emptyset$ for all $A \in M(x)$;

(ii) for any $n \in \mathbb{N}$ there exists $\gamma_{n}>0$ such that

$$
\max _{E \in \mathcal{E}_{n}}\left\|x-P_{E} x\right\|=1-\gamma_{n} .
$$

Proof. (i). Assume that $E \subseteq \mathbb{N}$ with $E \cap A \neq \emptyset$ for all $A \in M(x)$ such that $\left\|x-P_{E} x\right\|=1$. By Lemma 2.7 there exists $B \in M\left(x-P_{E} x\right)$. But $M\left(x-P_{E} x\right) \subseteq$ $M(x)$ since $\left\|x-P_{E} x\right\|=1$ and this gives us the contradiction $B \cap E=\emptyset$.

Any $E \in \mathcal{E}_{n}$ satisfies $E \cap A \neq \emptyset$ for all $A \in M(x)$ and $\mathcal{E}_{n}$ is finite, so (ii) follows from (i).

Let $X$ be a Banach space and $x \in S_{X}$. If $x$ is a delta-point, then for every slice $S$ with $x \in S$, we have that $x$ is at one end of a line segment in $S$ with length as close to 2 as we want. Suppose we replace the slice $S$ with a non-empty relatively weakly open subset $W$ of $B_{X}$ with $x \in W$. If $X$ has the Daugavet property, then $x$ is at one end of a line segment in $W$ with length as close to 2 as we want ([Shv00, Lemma 3]). Next we show that this is never the case if $X$ has a 1-unconditional basis.

Proposition 2.12. Let $X$ be a Banach space with 1-unconditional basis $\left(e_{i}\right)_{i \in \mathbb{N}}$. If $x \in S_{X}$, then there exist $\delta>0$ and a relatively weakly open subset $W$, with $x \in W$, such that $\sup _{y \in W}\|x-y\|<2-\delta$.

Proof. Assume that $x \in S_{X} \cap K_{X}$. Let $E=\bigcup_{A \in M(x)} A(1)$. By Lemma 2.11 there exists $\gamma_{1}>0$ such that $\max _{F \in \mathcal{E}_{1}}\left\|x-P_{F} x\right\|=1-\gamma_{1}$. Let $\delta=\gamma_{1} / 2$.

Let $W=\left\{y \in B_{X}:\left|e_{i}^{*}(x-y)\right|<\min _{k \in E} \frac{x_{k}}{2}, i \in E\right\}$. Then $x \in W$, and if $y \in$ $W$, then $y_{i} \geq \frac{x_{i}}{2}>0$ for all $i \in E$. Thus if $y \in W$ we have

$$
\left\{i \in \mathbb{N}: y_{i} \geq \frac{x_{i}}{2}\right\} \cap E=E \in \mathcal{E}_{1} .
$$

For any $y \in W$, we get that

$$
\|x-y\| \leq\left\|\frac{x}{2}\right\|+\left\|\frac{x}{2}-P_{E} \frac{x}{2}\right\|+\left\|P_{E} \frac{x}{2}-y\right\|<2-\delta,
$$

and we are done.

Let us remark a fun application of the above proposition.

Remark 2.13. Let $K$ be an infinite compact Hausdorff space. Then $C(K)$ does not have a 1-unconditional (or a 1-suppression unconditional) basis.

Let $f$ be a function which attains its norm on a limit point of $K$. Arguing similarly as in [AHLP20, Theorem 3.4] we may find a sequence of norm one functions 
$g_{k}$ with distance as close to 2 as we want from $f$ that converge pointwise, and thus weakly, to $f$. The conclusion follows from Proposition 2.12

The next result is the key ingredient in our proof that there are no delta-points in Banach spaces with subsymmetric bases. Its proof draws heavily upon Lemma 2.11.

Lemma 2.14. Let $X$ be a Banach space with 1-unconditional basis $\left(e_{i}\right)_{i \in \mathbb{N}}$ and let $x \in S_{X}$. Assume that there exists a slice $S\left(x^{*}, \delta\right)$, an $n \in \mathbb{N}$ and some $\eta>0$ such that

(i) $x \in S\left(x^{*}, \delta\right)$,

(ii) $y \in S\left(x^{*}, \delta\right)$ implies that

$$
\left\{i:\left|y_{i}\right|>\eta\left|x_{i}\right|, \operatorname{sgn} y_{i}=\operatorname{sgn} x_{i}\right\} \cap D(n) \neq \emptyset
$$

for all $D \in M^{\infty}(x)$.

Then $x$ is not a delta-point.

Proof. Assume that $x \in S_{X} \cap K_{X}$. Now for each $A \in \mathcal{F}_{n}$ find $x_{A}^{*} \in S_{X^{*}}$ such that $x_{A}^{*}\left(P_{A} x\right)=1$ with $x_{A}^{*}\left(e_{i}\right)=0$ for all $i \notin A$, and $x_{A}^{*}\left(e_{i}\right)>0$ for all $i \in A$. Let $z^{*}=\frac{1}{\left|\mathcal{F}_{n}\right|+1}\left(\sum_{A \in \mathcal{F}_{n}} x_{A}^{*}+x^{*}\right)$. Then $z^{*} \in B_{X^{*}}$ and

$$
\left\|z^{*}\right\| \geq z^{*}(x)>\frac{\left|\mathcal{F}_{n}\right|+1-\delta}{\left|\mathcal{F}_{n}\right|+1}=1-\frac{\delta}{\left|\mathcal{F}_{n}\right|+1} .
$$

For any $y \in S\left(z^{*},\left\|z^{*}\right\|-1+\frac{\delta}{\left|\mathcal{F}_{n}\right|+1}\right)$, we get that

$$
1-\frac{\delta}{\left|\mathcal{F}_{n}\right|+1}<\frac{1}{\left|\mathcal{F}_{n}\right|+1}\left(\sum_{A \in \mathcal{F}_{n}} x_{A}^{*}(y)+x^{*}(y)\right) \leq \frac{\left|\mathcal{F}_{n}\right|+x^{*}(y)}{\left|\mathcal{F}_{n}\right|+1} .
$$

Solving for $x^{*}(y)$ we get that

$$
1-\delta<x^{*}(y),
$$

and similarly $1-\delta<x_{A}^{*}(y)$. Thus, if $0<\eta<1-\delta$,

$$
F:=\left\{i: y_{i} \geq \eta x_{i}\right\} \bigcap\left(\bigcup_{E \in \mathcal{G}_{n}} E\right) \in \mathcal{E}_{n} .
$$

For any $y \in S\left(z^{*},\left\|z^{*}\right\|-1+\frac{\delta}{\left|\mathcal{F}_{n}\right|+1}\right)$ we now get from Lemma 2.11 that

$$
\begin{aligned}
\|x-y\| & \leq\left\|x-\eta P_{F} x\right\|+\left\|\eta P_{F} x-y\right\| \\
& \leq \eta\left\|x-P_{F} x\right\|+(1-\eta)\|x\|+1 \\
& \leq \eta \max _{E \in \mathcal{E}_{n}}\left\|x-P_{E} x\right\|+2-\eta \\
& \leq 2-\eta \gamma_{n}<2 .
\end{aligned}
$$

If $x \in S_{X}$ with $M^{\infty}(x)=\emptyset$ in the above lemma, then any slice $S\left(x^{*}, \delta\right)$ containing $x$ trivially satisfies Lemma 2.14 (ii), We record this in the following proposition.

Proposition 2.15. Let $X$ be a Banach space with 1-unconditional basis and let $x \in S_{X}$. If $M^{\infty}(x)=\emptyset$, then $x$ is not a delta-point.

We will also need the following lemma. 
Lemma 2.16. Let $X$ be a Banach space with 1-unconditional basis $\left(e_{i}\right)_{i \in \mathbb{N}}$. If $x \in K_{X}$, then for every $A \in M(x)$ and every $t>0$ we have $\left\|P_{A} x+t e_{i}\right\|>\|x\|$ for all $i \in A$.

Proof. Let $x \in K_{X}, A \in M(x)$ and $t>0$. Since $i \in A$ and $A \in M(x)$ we have $x_{i}>0$ and $\left\|P_{A} x-x_{i}\right\|<\|x\|=\left\|P_{A} x\right\|$. Put $\lambda=x_{i} /\left(t+x_{i}\right)$. Then $0<\lambda<1$ and $P_{A} x=\lambda\left(P_{A} x+t e_{i}\right)+(1-\lambda)\left(P_{A} x-x_{i} e_{i}\right)$, so

$$
\begin{aligned}
\|x\| & =\left\|P_{A} x\right\| \leq \lambda\left\|P_{A} x+t e_{i}\right\|+(1-\lambda)\left\|P_{A} x-x_{i} e_{i}\right\| \\
& <\lambda\left\|P_{A} x+t e_{i}\right\|+(1-\lambda)\left\|P_{A} x\right\| \\
& =\lambda\left\|P_{A} x+t e_{i}\right\|+(1-\lambda)\|x\|,
\end{aligned}
$$

and the conclusion follows.

Finally it is time to cash in some dividends and prove the main result of this section.

Theorem 2.17. If $X$ has subsymmetric basis $\left(e_{i}\right)_{i \in \mathbb{N}}$, then $X$ has no delta-points.

Proof. Assume $x \in S_{X} \cap K_{X}$. By Proposition 2.15 we may assume that $M^{\infty}(x) \neq \emptyset$. Let $s:=\max \left\{n: x_{n}=\max _{i} x_{i}\right\}$. We first show that $s \in A$ for all $A \in M^{\infty}(x)$.

For contradiction assume that there exists $A=\left\{a_{1}, a_{2}, \ldots\right\} \in M^{\infty}(x)$ with $s \notin A$. Let $a_{0}=0$ and $j \in \mathbb{N}$ be such that $a_{j-1}<s<a_{j}$. Let $t>0$ such that $x_{s}=x_{a_{j}}+t$ and let $A_{s}$ be $A$ with $a_{j}$ replaced by $s$. Using that $\left(e_{i}\right)_{i \in \mathbb{N}}$ is subsymmetric and Lemma 2.16 we get

$$
\begin{aligned}
1 \geq\left\|P_{A_{s}} x\right\| & =\left\|\sum_{i \neq j} x_{a_{i}} e_{i}+\left(x_{a_{j}}+t\right) e_{j}\right\| \\
& =\left\|\sum_{i \in \mathbb{N}} x_{a_{i}} e_{a_{i}}+t e_{a_{j}}\right\|=\left\|P_{A} x+t e_{a_{j}}\right\|>1
\end{aligned}
$$

a contradiction.

If we let $n=s$, then $s \in D(n)$ for all $D \in M^{\infty}(x)$, and the slice $S\left(e_{s}^{*}, 1-\frac{x_{s}}{2}\right)$ and $\eta=\frac{1}{2}$ satisfies the criteria in Lemma 2.14 and we are done.

In the proof above we saw that if $X$ has a subsymmetric basis, then for any $x \in S_{X}$ either $M^{\infty}(x)=\emptyset$ or all $A \in M^{\infty}(x)$ has a common element. In the case $X$ has a 1-symmetric basis we can say a lot about the sets $M(x)$ for any given $x \in S_{X}$.

Recall that a Schauder basis $\left(e_{i}\right)_{i \in \mathbb{N}}$ is called 1-symmetric if it is unconditional and $\left\|\sum_{i \in \mathbb{N}} \theta_{i} x_{i} e_{\pi(i)}\right\|=\left\|\sum_{i \in \mathbb{N}} x_{i} e_{i}\right\|$ for any $x=\sum_{i \in \mathbb{N}} x_{i} e_{i} \in X$, any sequence of signs $\left(\theta_{i}\right)_{i \in \mathbb{N}}$, and any permutation $\pi$ of $\mathbb{N}$. A 1 -symmetric basis is subsymmetric [LT77, Proposition 3.a.3].

Proposition 2.18. Let $X$ be a Banach space with 1-symmetric basis $\left(e_{i}\right)_{i \in \mathbb{N}}$ and let $x \in S_{X}$.

(i) If $M^{\infty}(x) \neq \emptyset$, then $M(x)=\{\operatorname{supp}(x)\}$;

(ii) If $M^{\infty}(x)=\emptyset$ and $A, B \in M(x)$, then $|A|=|B|$ and $x$ is constant on $A \triangle B$.

Proof. Assume that $x \in S_{X} \cap K_{X}$.

(i). Let $A \in M^{\infty}(x)$ and $x_{l} \in \operatorname{supp}(x) \backslash A$. Since $|A|=\infty$, there exists $k \in A$ and $t>0$ with $x_{k}+t=x_{l}$. Using that $\left(e_{i}\right)_{i \in \mathbb{N}}$ is 1 -symmetric and Lemma 2.16 we 
get

$$
1 \geq\left\|P_{A \backslash\{k\}} x+x_{l} e_{l}\right\|=\left\|P_{A \backslash\{k\}} x+x_{l} e_{k}\right\|=\left\|P_{A} x+t e_{k}\right\|>1,
$$

a contradiction.

(ii) Suppose that $x$ is not constant on $A \triangle B$ and let $k, l \in A \triangle B$ with $x_{k} \neq x_{l}$, say $k \in A, l \in B$, and $x_{k}<x_{l}$. Then argue as in (i) to get a contradiction, so $x$ is constant on $A \triangle B$. As $x$ is constant on $A \triangle B$, we cannot have $|A|<|B|$ since then a subset of $B$ would be in $M(x)$ contradicting the definition of $M(x)$.

\section{A SPACE WITH 1-UnCONDITIONAL BASIS AND DELTA-POINTS}

In this section we will prove the following theorem.

Theorem 3.1. There exists a Banach space $X_{\mathfrak{B}}$ with 1-unconditional basis, such that

(i) $X_{\mathfrak{B}}$ has a delta-point;

(ii) $X_{\mathfrak{B}}$ does not have Daugavet-points.

Before giving a proof of the theorem we will need some notation. By definition, a tree is a partially ordered set $(\mathcal{T}, \preceq)$ with the property that, for every $t \in \mathcal{T}$, the set $\{s \in \mathcal{T}: s \preceq t\}$ is well ordered by $\preceq$. In any tree we use normal interval notation, so that for instance a segment is $[s, t]=\{r \in \mathcal{T}: s \preceq r \preceq t\}$. If a tree has only one minimal member, it is said to be rooted and the minimal member is called the root of the tree and is denoted $\emptyset$. We have $\emptyset \preceq t$ for all $t \in \mathcal{T}$. We say that $t$ is an immediate successor of $s$ if $s \prec t$ and the set $\{r \in \mathcal{T}: s \prec r \prec t\}$ is empty. The set of immediate successors of $s$ we denote with $s^{+}$. A sequence $B=\left\{t_{n}\right\}_{n=0}^{\infty}$ is a branch of $\mathcal{T}$ if $t_{n} \in \mathcal{T}$ for all $n, t_{0}=\emptyset$ and $t_{n+1} \in t_{n}^{+}$for all $n \geq 0$. If $s, t \in \mathfrak{B}$ are nodes such that neither $s \preceq t$ nor $t \preceq s$, then $s$ and $t$ are incomparable. An antichain in a tree is a collection of elements which are pairwise incomparable.

We consider the infinite binary tree, $\mathfrak{B}=\bigcup_{n=0}^{\infty}\{0,1\}^{n}$, that is, finite sequences of zeros and ones. The order $\preceq$ on $\mathfrak{B}$ is defined as follows: If $s=\left\{s_{1}, s_{2}, \ldots, s_{k}\right\} \in$ $\{0,1\}^{k} \subset \mathfrak{B}$ and $t=\left\{t_{1}, t_{2}, \ldots, t_{l}\right\} \in\{0,1\}^{l} \subset \mathfrak{B}$, then $s \preceq t$ if and only if $k \leq l$ and $s_{i}=t_{i}, 1 \leq i \leq k$. As usual we denote with $|s|$ the cardinality of $s$, i.e. $|s|=k$. The concatenation of $s$ and $t$ is $s \frown t=\left\{s_{1}, s_{2}, \ldots, s_{k}, t_{1}, t_{2}, \ldots, t_{l}\right\} \in\{0,1\}^{k+l} \subset \mathfrak{B}$. Clearly $s \preceq s \frown t$ and $s^{+}=\{s \frown 0, s \frown 1\}$. The infinite binary tree is rooted with $\emptyset=\{0,1\}^{0}$. if

Following Talagrand [Tal79, Tal84] we say that $\mathcal{A} \subseteq \mathcal{P}(\mathbb{N})$ is an adequate family

- $\mathcal{A}$ contains the empty set and the singletons: $\{n\} \in \mathcal{A}$ for all $n \in \mathbb{N}$.

- $\mathcal{A}$ is hereditary: If $A \in \mathcal{A}$ and $B \subseteq A$, then $B \in \mathcal{A}$.

- $\mathcal{A}$ is compact with respect to the topology of pointwise convergence: Given $A \subset \mathbb{N}$, if every finite subset of $A$ is in $\mathcal{A}$, then $A \in \mathcal{A}$.

Given an adequate family $\mathcal{A}$, we define the Banach lattice $\ell_{\mathcal{A}, 1}$ as the set of all sequences $x=\left(a_{i}\right)_{i=1}^{\infty}$ satisfying $\|x\|=\sup _{A \in \mathcal{A}} \sum_{i \in A}\left|a_{i}\right|<\infty$ (see e.g. [AM93, Definition 2.1]). It is easy to see that, in general, the standard unit vectors $\left(e_{i}\right)_{i \in \mathbb{N}}$ form a normalized 1-unconditional basic sequence in $\ell_{\mathcal{A}, 1}$. We denote $h_{\mathcal{A}, 1}$ the closed subspace of $\ell_{\mathcal{A}, 1}$ generated by $\left(e_{i}\right)_{i \in \mathbb{N}}$. For example if $\mathcal{A}=\{\emptyset\} \cup\{\{n\}: n \in \mathbb{N}\}$, then $\ell_{\mathcal{A}, 1}=\ell_{\infty}, h_{\mathcal{A}, 1}=c_{0}$, and if $\mathbb{N} \in \mathcal{A}$, then $\ell_{\mathcal{A}, 1}=h_{\mathcal{A}, 1}=\ell_{1}$. Since $\mathcal{A}$ is compact we get that for every $x \in h_{\mathcal{A}, 1}$ there exists $A \in \mathcal{A}$ such that $\left\|P_{A} x\right\|=\|x\|$. 
There is a bijection between $\mathfrak{B}$ and $\mathbb{N}$ where the natural order on $\mathbb{N}$ corresponds to the lexicographical order on $\mathfrak{B}$ (see AT04, p. 69]). The family $\mathcal{A}$ of all subsets of $\mathbb{N}$ corresponding to the branches of $\mathfrak{B}$ and their subsets is an adequate family. We get that $X_{\mathfrak{B}}:=h_{\mathcal{A}, 1}$ is a Banach space with 1 -unconditional basis $\left(e_{t}\right)_{t \in \mathfrak{B}}$. It is worth pointing out that we use $t \in \mathfrak{B}$ as indices for the basis. Thus, for $x \in X_{\mathfrak{B}}$ and any non-negative integer $n$ we write $\sum_{|t|>n} e_{t}^{*}(x) e_{t}$, when referring to the sum $\sum_{t \in \mathfrak{B},|t|>n} e_{t}^{*}(x) e_{t}$, that is, $t \in \mathfrak{B}$ is implicit. A similar notation will be used in Section 4

Note that the span of the basis vectors corresponding to any infinite antichain in $X_{\mathfrak{B}}$ is isometric to $c_{0}$, and that the span of the basis vectors corresponding to any branch in $X_{\mathfrak{B}}$ is isometric to $\ell_{1}$.

Proof of Theorem 3.1)(i), Consider

$$
x=\sum_{|t|>0} 2^{-|t|} e_{t} .
$$

Summing over branches we find that $\|x\|=1$. We will show that $x$ is a delta-point. Define $z_{\emptyset}=0$ and then for $t_{0} \in \mathfrak{B}$

$$
z_{t_{0} 0}=z_{t_{0}}+e_{t_{0} 1} \text { and } z_{t_{0} 1}=z_{t_{0}}+e_{t_{\widehat{0}} 0} \text {. }
$$

Here is a picture of $z_{(0,0)}$ and $z_{(0,1)}$ :
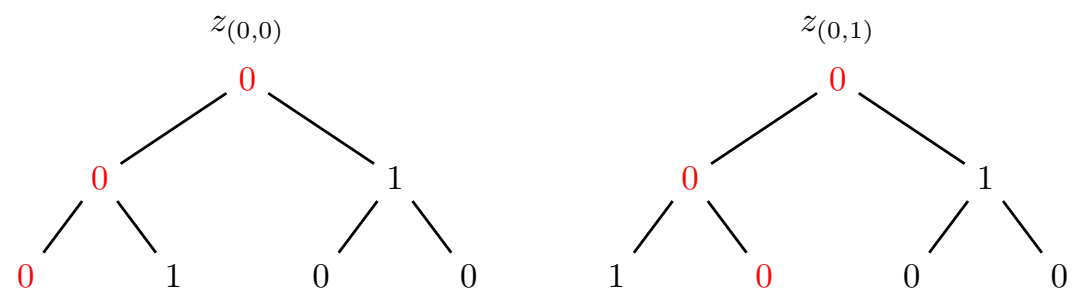

From the definition it is clear that

$$
\frac{1}{2}\left(z_{t_{0} 0}+z_{t_{0} 1}\right)=z_{t_{0}}+\frac{1}{2}\left(e_{t_{0} 0}+e_{t_{0}^{-1}}\right)
$$

so by induction

$$
y_{N}:=\frac{1}{2^{N}} \sum_{|t|=N} z_{t}=x-\sum_{|t|>N} 2^{-|t|} e_{t} .
$$

Let $x^{*} \in S_{X_{\mathfrak{B}}^{*}}$ and $\delta>0$ such that $x \in S\left(x^{*}, \delta\right)$. Find $N$ such that $x^{*}\left(y_{N}\right)>1-\delta$ which is possible since $\left\|\sum_{|t|>N} 2^{-|t|} e_{t}\right\| \rightarrow 0$ as $N \rightarrow \infty$. But $x^{*}\left(y_{N}\right)>1-\delta$ means that there exists $t_{0}$ with $\left|t_{0}\right|=N$ such that $x^{*}\left(z_{t_{0}}\right)>1-\delta$. Let $E=\left(t_{i}\right)_{i=1}^{\infty}$ be an infinite antichain of successors of $t_{0}$. Then $x^{*}\left(e_{t_{i}}\right) \rightarrow 0$ as $i \rightarrow \infty$. Find $t_{n}$ such that

$$
x^{*}\left(z_{t_{0}}-e_{t_{n}}\right)>1-\delta .
$$

By definition of $z_{t_{0}}$ we have $\left\{u \in \mathfrak{B}: u \preceq t_{0}\right\} \cap \operatorname{supp}\left(z_{t_{0}}\right)=\emptyset$ hence $z_{t_{0}}-e_{t_{n}} \in$ $S\left(x^{*}, \delta\right)$. Summing over a branch containing $t_{n}$ we get

$$
\left\|x-\left(z_{t_{0}}-e_{t_{n}}\right)\right\| \geq \sum_{h=1, h \neq\left|t_{n}\right|}^{\infty} 2^{-h}+2^{-\left|t_{n}\right|}+1=2
$$

as desired. 
Next is the proof that $X_{\mathfrak{B}}$ does not have Daugavet-points. We first need a general lemma about Daugavet-points.

Let $\left(e_{i}\right)_{i \in \mathbb{N}}$ be a 1-unconditional basis in a Banach spaces $X$. Define

$$
E_{X}=\left\{E \subset \mathbb{N}: \sum_{i \in E} e_{i} \in S_{X}\right\}
$$

Lemma 3.2. Let $X$ be a Banach space with 1-unconditional basis $\left(e_{i}\right)_{i \in \mathbb{N}}$. If $x \in S_{X}$ is a Daugavet-point, then $\left\|x-P_{E} x\right\|=1$ for all $E \in E_{X}$.

Proof. Assume $x \in S_{X} \cap K_{X}$ and that there exists $\eta>0$ and $E \in E_{X}$ such that $\left\|x-P_{E} x\right\|<1-\eta$.

Define $x^{*}=\frac{1}{|E|} \sum_{i \in E} e_{i}^{*} \in S_{X^{*}}$. Choose $\gamma>0$ such that $\max _{i \in E} \frac{e_{i}^{*}(x)}{2}<1-\gamma$. If $y \in S\left(x^{*}, \frac{\gamma}{|E|}\right)$, then it follows that $1-\gamma<e_{i}^{*}(y)$ for all $i \in E$ and

$$
\|x-y\| \leq\left\|x-P_{E} \frac{x}{2}\right\|+\left\|y-P_{E} \frac{x}{2}\right\|<2-\frac{\eta}{2},
$$

so $x$ is not a Daugavet-point.

Proof of Theorem 3.1 (ii), Assume $x \in S_{X_{\mathfrak{B}}} \cap K_{X_{\mathfrak{B}}}$. Let $E=\bigcup_{A \in M(x)} A(1)$. From Lemma 2.8 we see that $|E|$ is finite. Note that $E$ is an antichain. Indeed, assume $t_{0}, t_{1} \in E$ with $t_{0} \preceq t_{1}$ where $A(1)=\left\{t_{1}\right\}$ for some $A \in M(x)$. Then since $x \in K_{X_{\mathfrak{B}}}$ and

$$
1 \geq\left\|P_{A \cup\left\{t_{0}\right\}} x\right\| \geq \sum_{t \in A \cup\left\{t_{0}\right\}} e_{t}^{*}(x)=\sum_{t \in A \backslash\left\{t_{0}\right\}} e_{t}^{*}(x)+e_{t_{0}}^{*}(x) \geq\left\|P_{A} x\right\|=1
$$

we must have $t_{0}=t_{1}$.

We have $\left\|x-P_{E} x\right\|<1$ by Lemma 2.11)(i). From Lemma 3.2 we get that $x$ is not a Daugavet-point since $E \in E_{X_{\mathfrak{B}}}$.

Let us end this section with a remark about the proof of Theorem[3.1 (i) In order to prove that $X_{\mathfrak{B}}$ has a delta-point we could have used dyadic trees. Recall that a dyadic tree in a Banach space is a sequence $\left(x_{t}\right)_{t \in \mathfrak{B}}$, such that $x_{t}=\frac{1}{2}\left(x_{t-0}+x_{t-1}\right)$.

In fact, $x=\sum_{|t|>0} 2^{-|t|} e_{t}$ is the root of a dyadic tree. In order to show this one uses the same $z_{t}$ 's as in the above proof, but attach a copy of $x$ to the node $t$. Finally, we have the following result about dyadic trees and delta-points.

Proposition 3.3. If a Banach space $X$ contains a dyadic tree $\left(x_{t}\right)_{t \in \mathfrak{B}} \subset B_{X}$ such that

$$
\limsup _{n \rightarrow \infty}\left(\min _{|t|=n}\left\{\left\|x_{\emptyset}-x_{t}\right\|\right\}\right)=2
$$

then $x_{\emptyset}$ is a delta-point.

Proof. Let $\varepsilon>0$ and find $n$ with $\left\|x_{\emptyset}-x_{t}\right\| \geq 2-\varepsilon$ for all $t$ with $|t|=n$. This means that $x_{t} \in \Delta_{\varepsilon}\left(x_{\emptyset}\right)$. By definition of a dyadic tree

$$
x_{\emptyset}=\frac{1}{2^{n}} \sum_{|t|=n} x_{t}
$$

so we have $x_{\emptyset} \in \operatorname{conv} \Delta_{\varepsilon}\left(x_{\emptyset}\right)$. 


\section{A SPACE With 1-UnCONDItional Basis AND DAUGAVET-POINTS}

In this section we will cut of the root of the binary tree and modify the norm from the example in the previous section to allow the space to have Daugavet-points.

Let $\mathfrak{M}=\bigcup_{n=1}^{\infty}\{0,1\}^{n}$ be the binary tree with the root removed. Note that a branch $B=\left\{t_{n}\right\}_{n=1}^{\infty}$ in $\mathfrak{M}$ corresponds to the branch $\left\{t_{n}\right\}_{n=0}^{\infty}$ in $\mathfrak{B}$ where $t_{0}=\emptyset$.

A $\lambda$-segment in $\mathfrak{M}$ is a set $S \subset \mathfrak{M}$ of the form $S=[s, t] \cup t^{+}$, where $[s, t]$ is a (possibly empty) segment of $\mathfrak{M}$. If $[s, t]=\emptyset$, then $S=\{(0),(1)\}$.

Using the lexicographical order $\leq$ on $\mathfrak{M}$ we have a bijective correspondence to $\mathbb{N}$ with the natural order. Let $\mathcal{A}$ be the adequate family of subsets of $\mathbb{N}$ corresponding to subsets of branches and subsets of $\lambda$-segments. Using this adequate family we get a Banach space $X_{\mathfrak{M}}:=h_{\mathcal{A}, 1}$ with 1 -unconditional basis $\left(e_{t}\right)_{t \in \mathfrak{M}}$. We call $X_{\mathfrak{M}}$ the modified binary tree space. Note that $X_{\mathfrak{M}}$ contains isometric copies of $c_{0}$ and $\ell_{1}$ just like $X_{\mathfrak{B}}$.

As we saw in the proof of Theorem 3.1) (ii) the antichains in the tree play an important role for the existence of Daugavet-points.

Define

$$
\mathfrak{F}:=\{0\} \cup\left\{z \in S_{X_{\mathfrak{M}}}: z(\mathfrak{M}) \subset\{0, \pm 1\}\right\} .
$$

The set $E_{X_{\mathfrak{M}}}$ from Section 3 can be described as the set of all non-void finite antichains $E$ of $\mathfrak{M}$ such that $|A \cap E| \leq 1$ for all $A \in \mathcal{A}$. Clearly $\operatorname{supp}(z) \in E_{X_{\mathfrak{M}}}$ for every $z \in \mathfrak{F} \backslash\{0\}$ and every $z$ with $\operatorname{supp}(z) \in E_{X_{\mathfrak{M}}}$ and $z(\mathfrak{M}) \subset\{0, \pm 1\}$ belongs to $\mathfrak{F}$. It is also clear that for every $E \in E_{X_{\mathfrak{M}}}$ there exists a branch $B$ such that $B \cap E=\emptyset$. We will see in Lemma 4.1 and Theorem 4.2 that the sets $E_{X_{\mathfrak{M}}}$ and $\mathfrak{F}$ will play an essential role in characterizing the Daugavet-points of $X_{\mathfrak{M}}$.

If $M$ is a finite subset of $\mathfrak{M}$, then we will use the notation $K_{M}=\left\{\sum_{t \in M} a_{t} e_{t}\right.$ : $\left.a_{t} \geq 0\right\}$ and $\mathfrak{F}_{M}=\{z \in \mathfrak{F}: \operatorname{supp}(z) \subset M\}$.

First we prove a lemma which says that convex combinations of elements in $\mathfrak{F}$ are dense in the unit ball of $X_{\mathfrak{M}}$.

Lemma 4.1. Let $M$ be a finite subset of $\mathfrak{M}$. Then

$$
\operatorname{span}\left\{e_{t}: t \in M\right\} \cap B_{X_{\mathfrak{M}}}=\operatorname{conv}\left(\mathfrak{F}_{M}\right)
$$

that is, for every $x \in \operatorname{span}\left\{e_{t}: t \in M\right\} \cap B_{X_{\mathfrak{M}}}$ we have

$$
x=\sum_{k=1}^{N} \lambda_{k} z_{k}
$$

where $z_{k} \in \mathfrak{F}_{M}, \lambda_{k}>0, \sum_{k=1}^{N} \lambda_{k}=1$. In particular, $\operatorname{ext}\left(K_{M} \cap B_{X_{\mathfrak{M}}}\right)=K_{M} \cap \mathfrak{F}_{M}$.

Proof. With $M_{n}$ denote the subset of $\mathfrak{M}$ which corresponds to $\{1, \ldots, n\} \subset \mathbb{N}$. We will show, by induction, that for every $x \in K_{M_{2 n}} \cap B_{X_{\mathfrak{M}}}$ we have

$$
x=\sum_{k=1}^{N} \lambda_{k} z_{k},
$$

where $z_{k} \in K_{\operatorname{supp}(x)} \cap \mathfrak{F}, \lambda_{k}>0$ and $\sum_{k=1}^{N} \lambda_{k}=1$. As $K_{M} \subseteq K_{M_{2 n}}$ for some $n \in \mathbb{N}$ and $z_{k} \in K_{\operatorname{supp}(x)} \cap \mathfrak{F}$, the result will follow. 
The base step is $x \in K_{M_{2}} \cap B_{X_{\mathfrak{M}}}$ with $e_{t}^{*}(x) \geq 0$ for $t \in M_{2}=\{(0),(1)\}$. Write $e_{(0)}^{*}(x)=a_{0}$ and $e_{(1)}^{*}(x)=a_{1}$. Define $c=1-a_{0}-a_{1}, z_{0}=e_{(0)}$, and $z_{1}=e_{(1)}$. Then

$$
x=\left(c \cdot 0+a_{0} z_{0}+a_{1} z_{1}\right)
$$

is a convex combination of elements in $K_{\operatorname{supp}(x)} \cap \mathfrak{F}$.

Assume the induction hypothesis holds for $n \in \mathbb{N}$. Let $x \in K_{M_{2(n+1)}} \cap B_{X_{\mathfrak{M}}}$. Let $t \in \mathfrak{M}$ be the node such that $t \frown 0$ corresponds to $2 n+1$ and $t^{\frown} 1$ to $2 n+2$. Define

$$
x^{\prime}=x-e_{t-0}^{*}(x) e_{t \frown 0}-e_{t-1}^{*}(x) e_{t \frown 1} .
$$

By assumption we have $x^{\prime}=\sum_{k=1}^{N} \lambda_{k} z_{k}$ with $\lambda_{k}>0, \sum_{k=1}^{N} \lambda_{k}=1$ and $z_{k} \in$ $K_{\text {supp }\left(x^{\prime}\right)} \cap \mathfrak{F}$.

Define the segment $A=\{s \in \mathfrak{M}: s \preceq t\}$ and the sets

$$
I=\left\{k \in\{1, \ldots, N\}: P_{A} z_{k}=0\right\} \quad \text { and } \quad J=\{1, \ldots, N\} \backslash I .
$$

For $k \in I$ we let

$$
z_{k, 0}:=z_{k}+e_{t \frown 0} \quad \text { and } \quad z_{k, 1}:=z_{k}+e_{t \frown 1} .
$$

Since $z_{k} \in K_{\operatorname{supp}\left(x^{\prime}\right)} \cap \mathfrak{F}$ we get $z_{k, 0}, z_{k, 1} \in K_{\operatorname{supp}(x)} \cap \mathfrak{F}$ and

$$
\sum_{s \in A} e_{s}^{*}\left(x^{\prime}\right)=\sum_{s \in A} e_{s}^{*}(x)=\sum_{k \in J} \lambda_{k}
$$

Thus, by definition of the norm we have,

$$
0 \leq e_{t-0}^{*}(x)+e_{t-1}^{*}(x) \leq 1-\sum_{s \in A} e_{s}^{*}(x)=\sum_{k \in I} \lambda_{k} .
$$

Write $e_{t-0}^{*}(x)=a_{0}$ and $e_{t-1}^{*}(x)=a_{1}$. Define $c=\sum_{k \in I} \lambda_{k}-a_{0}-a_{1}$. Let $m=$ $\sum_{k \in I} \lambda_{k}$. It follows that

$$
\begin{aligned}
x & =x^{\prime}+a_{0} e_{t \frown 0}+a_{1} e_{t-1} \\
& =\sum_{k \in J} \lambda_{k} z_{k}+\sum_{k \in I} \lambda_{k} z_{k}+\sum_{k \in I} \lambda_{k}\left(\frac{a_{0}}{m} e_{t \frown 0}+\frac{a_{1}}{m} e_{t \frown 1}\right) \\
& =\sum_{k \in J} \lambda_{k} z_{k}+\sum_{k \in I} \lambda_{k} \frac{\left(a_{0}+a_{1}+c\right)}{m} z_{k}+\sum_{k \in I} \lambda_{k}\left(\frac{a_{0}}{m} e_{t \frown 0}+\frac{a_{1}}{m} e_{t \frown 1}\right) \\
& =\sum_{k \in J} \lambda_{k} z_{k}+\sum_{k \in I} \lambda_{k}\left(\frac{a_{0}}{m} z_{k, 0}+\frac{a_{1}}{m} z_{k, 1}+\frac{c}{m} z_{k}\right)
\end{aligned}
$$

which is a convex combination of elements in $K_{\operatorname{supp}(x)} \cap \mathfrak{F}$.

With the above lemma in hand we are able to characterize Daugavet-points in $X_{\mathfrak{M}}$ in terms of $E_{X_{\mathfrak{M}}}$. This will give us an easy way to identify and give examples of Daugavet-points.

Theorem 4.2. Let $x \in S_{X_{\mathfrak{M}}}$, then the following are equivalent

(i) $x$ is a Daugavet-point;

(ii) $\left\|x-P_{E} x\right\|=1$, for all $E \in E_{X_{\mathfrak{M}}}$;

(iii) for any $z \in \mathfrak{F}$, either $\|x-z\|=2$ or for all $\varepsilon>0$ there exists $s \in \mathfrak{M}$ such that $z \pm e_{s} \in \mathfrak{F}$ and $\left\|x-z \pm e_{s}\right\|>2-\varepsilon$. 
Proof. As usual we will assume that $x \in K_{X_{\mathfrak{M}}}$ throughout.

(i) $\Rightarrow$ (ii) is Lemma 3.2

(ii) $\Rightarrow$ (iii). Let $\varepsilon>0, z \in \mathfrak{F}$ and $E=\operatorname{supp}(z)$. We have assumed that $\left\|x-P_{E} x\right\|=1$.

By definition of $M\left(x-P_{E} x\right)$ we have $A \cap E=\emptyset$ for every $A \in M\left(x-P_{E} x\right)$. If there, for some $A \in M\left(x-P_{E} x\right)$, exists $t \in E$ and $s_{0} \in A$ such that $t \preceq s_{0}$, or $t \in E$ such that $s \preceq t$ for all $s \in A$, then we are done since $e_{t}^{*}(x)=0$ and

$$
\|x-z\| \geq \sum_{s \in A}\left|e_{s}^{*}(x)\right|+\left|e_{t}^{*}(z)\right|=2 .
$$

So from now on we assume that no such $A$ exists.

Assume that there exists $A \in M\left(x-P_{E} x\right)$ that is a subset of a branch $B$. By definition of the norm, we have $e_{t}^{*}(x)=0$ for $t \in B \backslash A$, and by the assumption above, we also have $B \cap E=\emptyset$. Since $\left|e_{t}^{*}(x)\right| \rightarrow 0$ as $|t| \rightarrow \infty$ for $t \in B$ we can find $s \in B$ with $\left|e_{s}^{*}(x)\right|<\varepsilon / 2$ and hence

$$
\left\|x-z \pm e_{s}\right\| \geq \sum_{t \in A, t \neq s}\left|e_{t}^{*}(x)\right|+\left|e_{s}^{*}(x) \pm 1\right| \geq 2-\varepsilon .
$$

This concludes the case where $A$ is a subset of a branch.

Suppose for contradiction that no $A \in M\left(x-P_{E} x\right)$ is a subset of a branch, then every $B \in M\left(x-P_{E} x\right)$ is a subset of a $\lambda$-segment. By Lemma 2.10 we must have $\left|M\left(x-P_{E} x\right)\right|<\infty$.

Choose any $B \in M\left(x-P_{E} x\right)$ and write

$$
B=\left\{b_{1} \prec b_{2} \prec \cdots \prec b_{n}\right\} \cup\{b \frown 0, b \frown 1\},
$$

where $b_{n} \preceq b$. In particular $e_{s}^{*}(x) \neq 0$ for $s \in b^{+}$.

Let $R=\{t \in E: b \frown 0 \prec t\}$ and $E_{1}=(E \cup\{b \frown 0\}) \backslash R$. From the assumptions above $E \cap\{t: t \preceq b \frown 0\}=\emptyset$, so $E_{1} \in E_{X_{\mathfrak{M}}}$.

Let $C \in M\left(x-P_{E_{1}} x\right)$. Notice that $C \cap\{t: b \frown 0 \preceq t\}=\emptyset$. Otherwise, by definition of the norm, we get the contradiction

$$
1=\left\|P_{C \cap\{b \frown 0\}} x\right\|=\sum_{t \in C}\left|e_{t}^{*}(x)\right|+\left|e_{b \frown 0}^{*}(x)\right|>\sum_{t \in C}\left|e_{t}^{*}(x)\right|=\left\|P_{C} x\right\|=1 .
$$

Hence $P_{C}\left(x-P_{E_{1}} x\right)=P_{C}\left(x-P_{E} x\right)$ and $C \in M\left(x-P_{E} x\right)$.

We have $M\left(x-P_{E_{1}} x\right) \subseteq M\left(x-P_{E} x\right)$, but since $B \cap E_{1} \neq \emptyset$ we have $B \notin$ $M\left(x-P_{E_{1}} x\right)$ so the inclusion is strict.

We now have $\left|M\left(x-P_{E_{1}} x\right)\right|<\left|M\left(x-P_{E} x\right)\right|$ and no $C \in M\left(x-P_{E_{1}} x\right)$ is a subset of a branch. We can use the argument above a finite number of times until we are left with $E_{m} \in E_{X_{\mathfrak{M}}}$ with $\left\|x-P_{E_{m}} x\right\|=1$ and $M\left(x-P_{E_{m}} x\right)=\emptyset$ which contradicts Lemma 2.7

Finally, (iii) $\Rightarrow$ (i). Choose $\varepsilon>0$. Let $y \in B_{X_{\mathfrak{M}}}$ with finite support. Then by Lemma 4.1, we can write $y=\sum_{k=1}^{n} \lambda_{k} z_{k}$, with $z_{k} \in \mathfrak{F}, \lambda_{k} \geq 0$ and $\sum_{k=1}^{n} \lambda_{k}=1$. Let $D_{1}=\left\{k \in\{1, \ldots, n\}:\left\|x-z_{k}\right\|=2\right\}$ and $D_{2}=\{1, \ldots, n\} \backslash D_{1}$. We can, by assumption, for each $k \in D_{2}$ find $s_{k} \in \mathfrak{M}$ such that $z_{k} \pm e_{s_{k}} \in \mathfrak{F}$ with $\left\|x-z_{k} \pm e_{s_{k}}\right\|>2-\varepsilon$. Then $y \in \operatorname{conv} \Delta_{\varepsilon}(x)$ since

$$
y=\sum_{i \in D_{1}} \lambda_{k} z_{k}+\sum_{k \in D_{2}} \frac{\lambda_{k}}{2}\left(z_{k}+e_{s_{k}}\right)+\sum_{k \in D_{2}} \frac{\lambda_{k}}{2}\left(z_{k}-e_{s_{k}}\right) .
$$

The set of all such $y$ is dense in $B_{X_{\mathfrak{M}}}$, hence $B_{X_{\mathfrak{M}}}=\overline{\operatorname{conv}} \Delta_{\varepsilon}(x)$ so $x$ is a Daugavetpoint. 
Corollary 4.3. If $x \in S_{X_{\mathfrak{M}}}$ such that $\left\|P_{A} x\right\|=1$ for all branches $A$, then $x$ is a Daugavet-point.

Proof. Let $E \in E_{X_{\mathfrak{M}}}$. There exists a branch $B$ such that $B \cap E=\emptyset$. Then $\left\|x-P_{E} x\right\| \geq\left\|P_{B} x\right\|=1$. By Theorem $4.2 x$ is a Daugavet-point.

With a characterization of Daugavet-points in hand we can now prove the main result of this section.

Theorem 4.4. In $X_{\mathfrak{M}}$ we have that

(i) there exists $x \in S_{X_{\mathfrak{M}}}$ which is a Daugavet-point;

(ii) there exists $w \in S_{X_{\mathfrak{M}}}$ which is a delta-point, but not a Daugavet-point.

Proof. Let $x=\sum_{t \in \mathfrak{M}} 2^{-|t|} e_{t}$. We have that $x$ is a Daugavet-point by Corollary 4.3 ,

The next part of the proof is similar to the proof of Theorem 3.1 (i) We will show that a shifted version of $x$ is a delta-point which is not a Daugavet-point. Define an operator on the modified binary tree:

$$
L\left(\sum_{|t|>0} a_{t} e_{t}\right)=\sum_{|t| \geq 0} a_{0 \frown{ }_{t}} e_{0}-t+\sum_{|t| \geq 0} a_{1-{ }_{t}} e_{(1,0)-t},
$$

where $t=\emptyset$ when $|t|=0$.

Define $w=L(x)$. Let $x^{*} \in S_{X_{\mathfrak{M}}^{*}}$ and $\delta>0$ such that $w \in S\left(x^{*}, \delta\right)$. Just as in the proof of Theorem 3.1] (i) we can find $z_{t_{0}} \in S_{X_{\mathfrak{M}}}$ whose support is an antichain (i.e. $\left.z_{t_{0}} \in \mathfrak{F}\right)$ and we can find $e_{t_{n}}$ such that $z_{t_{0}}-e_{t_{n}} \in S\left(x^{*}, \delta\right)$. Summing over a branch containing $t_{n}$ we get $\left\|w-\left(z_{t_{0}}-e_{t_{n}}\right)\right\|=2$.

Let $E=\{(0),(1,0)\}$. Then $\left\|w-P_{E} w\right\|=\sum_{i=2}^{\infty} 2^{-i}=\frac{1}{2}<1$ so by Theorem 4.2 $w$ is not a Daugavet-point.

In [AHLP20], the property that the unit ball of a Banach space is the closed convex hull of its delta-points was studied. We will next show that $X_{\mathfrak{M}}$ satisfies something much stronger, the unit ball is the closed convex hull of a subset of its Daugavet-points.

If $D$ is the set of all Daugavet-points in $X_{\mathfrak{M}}$ define

$$
D_{B}=\left\{x \in D:\left\|P_{B} x\right\|=1 \text { for all branches } B \text { of } \mathfrak{M}\right\} .
$$

The proof of Theorem 4.4 shows that $D_{B}$ is non-empty. is

For $t_{0} \in \mathfrak{M}$, let $S_{t_{0}}$ be the shift operator on $X_{\mathfrak{M}}$ that shifts the root to $t_{0}$, that

$$
S_{t_{0}}\left(\sum_{t \in \mathfrak{M}} a_{t} e_{t}\right)=\sum_{t \in \mathfrak{M}} a_{t} e_{t_{0}} t
$$

It is clear that $S_{t_{0}}$ is an isometry on $X_{\mathfrak{M}}$.

Proposition 4.5. The space $X_{\mathfrak{M}}$ satisfies $B_{X_{\mathfrak{M}}}=\overline{\operatorname{conv}}\left(D_{B}\right)$.

Proof. Let $y \in B_{X_{\mathfrak{M}}}$. We may assume that $y$ has finite support, since such $y$ are dense in $B_{X_{\mathfrak{M}}}$. By Lemma 4.1, we can write $y=\sum_{k=1}^{n} \lambda_{k} z_{k}$ where $z_{k} \in \mathfrak{F}, \lambda_{k} \geq 0$ and $\sum_{k=1}^{n} \lambda_{k}=1$.

Fix $z \in \mathfrak{F}$. Let $m:=\max \{|t|: t \in \operatorname{supp}(z)\}$.

$$
\mathcal{B}=\left\{t \in \mathfrak{M}:|t|=m, \sum_{s \preceq t}\left|e_{s}^{*}(z)\right|=0\right\} .
$$


Choose any $x_{0} \in D_{B}$ and use the shift operator in (2) to define

$$
x:=\sum_{t \in \mathcal{B}} S_{t}\left(x_{0}\right) .
$$

Observe that $z \pm x$ takes its norm along every branch, so by Corollary 4.3 both $z \pm x \in D_{B}$.

Repeat this construction for $z_{k}$ to create $x_{k}$ for $k \in\{1, \ldots, n\}$. Then

$$
y=\sum_{k=1}^{n} \frac{\lambda_{k}}{2}\left(z_{k}+x_{k}\right)+\sum_{k=1}^{n} \frac{\lambda_{k}}{2}\left(z_{k}-x_{k}\right),
$$

is a convex combination of Daugavet-points in $D_{B}$.

Our next result is that $X_{\mathfrak{M}}$ has the remarkable property that the Daugavet-points are weakly dense in the unit ball. So in a sense there are lots of Daugavet-points, but of course not enough of them in order for $X_{\mathfrak{M}}$ to have the Daugavet property. First we need a lemma. For $t \in \mathfrak{M}, S_{t}$ denotes the shift operator defined in (2) above.

Lemma 4.6. Let $x^{*} \in S_{X_{\mathfrak{M}}^{*}}$ and $s \in \mathfrak{B}$. For any $x \in S_{X_{\mathfrak{M}}}$ and $\varepsilon>0$ there exist some infinite antichain $E=\left\{t_{i}\right\}_{i=1}^{\infty}$ with the following properties

(i) $\left\|\sum_{i=1}^{n} e_{t_{i}}\right\|=1$ for all $n \in \mathbb{N}$;

(ii) $s \preceq t$ for all $t \in E$;

(iii) $\left|x^{*}\left(S_{t} x\right)\right|<\varepsilon$ for all $t \in E$.

Proof. Pick any $x^{*} \in S_{X_{\mathfrak{M}}^{*}}, s \in \mathfrak{B}$ and $x \in S_{X_{\mathfrak{M}}}$. It is not difficult to find an infinite antichain $E=\left\{t_{i}\right\}_{i=1}^{\infty}$ satisfying (i) and (ii). Since $E$ is an antichain we have $\left\|\sum_{i=1}^{n} S_{t_{i}}(x)\right\|=1$ for all $n \in \mathbb{N}$. Hence

$$
\lim _{i \rightarrow \infty} x^{*}\left(S_{t_{i}} x\right)=0,
$$

and then we can find $n \in \mathbb{N}$ such that $\left|x^{*}\left(S_{t_{i}} x\right)\right|<\varepsilon$ for all $i \geq n$. Now $E^{\prime}=$ $E \backslash\left\{t_{i}\right\}_{i=1}^{n}$ satisfies (i) (ii) and (iii)

Theorem 4.7. In $X_{\mathfrak{M}}$ every non-empty relatively weakly open subset of $B_{X_{\mathfrak{M}}}$ contains a Daugavet-point.

Proof. Since vectors with finite support are norm dense in $B_{X_{\mathfrak{M}}}$, it enough show that for any $y \in B_{X_{\mathfrak{M}}}$ with finite support and any relatively weakly open neighbourhood of $y$ of the form

$$
W:=\left\{x \in B_{X_{\mathfrak{m}}}:\left|x_{i}^{*}(y-x)\right|<\varepsilon, i=1, \ldots, n\right\},
$$

where $x_{i}^{*} \in S_{X_{\mathfrak{M}}^{*}}, i=1, \ldots, n$ and $\varepsilon>0$, contains a Daugavet-point.

Let $m:=\max \{|t|: t \in \operatorname{supp}(y)\}$, and for $t \in \mathfrak{M}$ with $|t|=m$ define

$$
\mu_{t}:=1-\sum_{s \preceq t}\left|e_{s}^{*}(y)\right|
$$

and

$$
\mathcal{N}:=\left\{t \in \mathfrak{M}:|t|=m, \mu_{t}>0\right\} .
$$

From Corollary 4.3 we have that $g=\sum_{s \in \mathfrak{M}} 2^{-|s|} e_{s}$ is a Daugavet-point. By Lemma 4.6 for each $t \in \mathcal{N}$ there exists $t \preceq b_{t}$ such that $\left|x_{i}^{*}\left(S_{b_{t}} g\right)\right|<\varepsilon / 2^{m}$ for $i=1, \ldots, n$. Now put

$$
x=y+\sum_{t \in \mathcal{N}} \mu_{t} S_{b_{t}}(g) .
$$


By construction $x \in S_{X_{\mathfrak{M}}}$ and we have $x \in W$ since

$$
\left|x_{i}^{*}(y-x)\right|=\left|x_{i}^{*}\left(\sum_{t \in \mathcal{N}} \mu_{t} S_{b_{t}}(g)\right)\right| \leq \sum_{t \in \mathcal{N}} \mu_{t}\left|x_{i}^{*}\left(S_{b_{k}} g\right)\right|<\frac{\varepsilon}{2^{m}} \sum_{t \in \mathcal{N}} \mu_{t}<\varepsilon .
$$

Using Theorem 4.2 we will show that $x$ is a Daugavet-point. Indeed, let $E \in E_{X_{\mathfrak{M}}}$. Then there exists a branch $A$ with $A \cap E=\emptyset$. Let $t \in A$ with $|t|=m$. If $t \notin \mathcal{N}$, then

$$
\left\|x-P_{E} x\right\| \geq \sum_{s \preceq t}\left|e_{s}^{*}(y)\right|=1 .
$$

If $t \in \mathcal{N}$, then since $S_{b_{t}}(g)$ is a Daugavet-point, there exists a branch $B$ with $t \in B$ such that $\left\|S_{b_{t}}(g)-P_{E} S_{b_{t}}(g)\right\|=\sum_{s \in B}\left|S_{b_{t}}(g)_{s}\right|=1$. Thus

$$
\left\|x-P_{E} x\right\| \geq \sum_{s \preceq t}\left|e_{s}^{*}(y)\right|+\sum_{\substack{s \in B \\ s \succ b_{t}}} \mu_{t}\left|S_{b_{t}}(g)_{s}\right|=1-\mu_{t}+\mu_{t}=1,
$$

and we are done.

Question 4.8. How "massive" does the set of Daugavet-points in $S_{X}$ have to be in order to ensure that a Banach space $X$ fails to have an unconditional basis?

If $S$ is a slice of the unit ball of $X_{\mathfrak{M}}$, then the above proposition tells us that $S$ contains a Daugavet-point $x$. Then by definition of Daugavet-points there exists for any $\varepsilon>0$ a $y \in S$ with $\|x-y\| \geq 2-\varepsilon$. Thus the diameter of every slice of the unit ball of $X_{\mathfrak{M}}$ is 2 , that is $X_{\mathfrak{M}}$ has the local diameter two property.

The next natural question is whether the diameter of every non-empty relatively weakly open neighborhood in $B_{X_{\mathfrak{M}}}$ equals 2 , that is, does $X_{\mathfrak{M}}$ have the diameter two property? The answer is no, in fact, every Daugavet-point in $D_{B}$ has a weak neighborhood of arbitrary small diameter. Let us remark that the first example of a Banach space with the local diameter two property, but failing the diameter two property was given in BGLPRZ15. While we have used binary trees, their construction used the tree of finite sequences of positive integers and they even showed that every Banach space containing $c_{0}$ can be renormed to have the local diameter two property and fail the diameter two property.

Proposition 4.9. In $X_{\mathfrak{M}}$ every $x \in D_{B}$ is a point of weak-to norm-continuity for the identity map on $B_{X_{\mathfrak{M}}}$. In particular, $X_{\mathfrak{M}}$ fails the diameter two property.

Proof. Let $\varepsilon>0$ and $x \in D_{B}$. Let $n \in \mathbb{N}$ be such that $\left\|\sum_{|t|>n} x_{t} e_{t}\right\|<\frac{\varepsilon}{8}$. Consider the weak neighborhood $W$ of $x$

$$
W=\left\{y \in B_{X_{\mathfrak{M}}}:\left|e_{t}^{*}(x-y)\right|<\frac{\varepsilon}{2^{|t|+3}},|t| \leq n\right\} .
$$

We want to show that the diameter of $W$ is less than $\varepsilon$. Let $y=\sum_{t \in \mathfrak{M}} y_{t} e_{t} \in W$. Let $A$ be a subset of a branch or of a $\lambda$-segment in $\mathfrak{M}$. Since $\left|x_{t}-y_{t}\right|<\varepsilon 2^{-|t|-3}$ for $|t| \leq n,\left\|\sum_{|t|>n} x_{t} e_{t}\right\|<\frac{\varepsilon}{8}$, and $x$ attains its norm along every branch of $\mathfrak{M}$, we have

$$
\sum_{\substack{t \in A \\|t| \leq n}}\left|y_{t}\right|>\sum_{\substack{t \in A \\|t| \leq n}}\left|x_{t}\right|-\left|x_{t}-y_{t}\right|>\sum_{\substack{t \in A \\|t| \leq n}}\left|x_{t}\right|-\frac{\varepsilon}{8}>1-\frac{\varepsilon}{4}
$$


Hence $\sum_{\substack{t \in A \\|t|>n}}\left|y_{t}\right|<\frac{\varepsilon}{4}$, and thus

$$
\begin{aligned}
\sum_{t \in A}\left|x_{t}-y_{t}\right| & =\sum_{\substack{t \in A \\
|t| \leq n}}\left|x_{t}-y_{t}\right|+\sum_{\substack{t \in A \\
|t|>n}}\left|x_{t}-y_{t}\right| \\
& <\sum_{\substack{t \in A \\
|t| \leq n}} \varepsilon 2^{-|t|-3}+\sum_{\substack{t \in A \\
|t|>n}}\left|x_{t}\right|+\sum_{\substack{t \in A \\
|t|>n}}\left|y_{t}\right| \\
& <\frac{\varepsilon}{8}+\frac{\varepsilon}{8}+\frac{\varepsilon}{4}=\frac{\varepsilon}{2} .
\end{aligned}
$$

From this it follows that the diameter of $W$ is less than $\varepsilon$.

Recall from [ALL16] that a Banach space $X$ is locally almost square if for every $x \in S_{X}$ and $\varepsilon>0$ there exists $y \in S_{X}$ such that $\|x \pm y\| \leq 1+\varepsilon$.

It is known that every locally almost square Banach space $X$ has the local diameter two property. As noted above $X_{\mathfrak{M}}$ has the local diameter two property, but it is not locally almost square as the following proposition shows.

Proposition 4.10. $X_{\mathfrak{M}}$ is not locally almost square.

Proof. Consider $x=\frac{1}{4} e_{(0)}+\frac{3}{4} e_{(1)}$. Let $0<\varepsilon<\frac{1}{4}$ and suppose there exists $y=\sum_{t \in \mathfrak{M}} y_{t} e_{t} \in S_{X_{\mathfrak{M}}}$ with $\|x \pm y\| \leq 1+\varepsilon<\frac{5}{4}$. Then clearly $\left|y_{(1)}\right| \leq \frac{1}{4}+\varepsilon$. By considering $-y$ if necessary we may assume that $y_{(1)} \geq 0$. Then

$$
\begin{aligned}
1+\varepsilon & \geq \max _{ \pm}\left\{\left|\frac{1}{4} \pm y_{(0)}\right|+\left|\frac{3}{4} \pm y_{(1)}\right|\right\} \\
& \geq\left|\frac{1}{4}-y_{(0)}\right|+\frac{3}{4}+\left|y_{(1)}\right| \\
& \geq\left|y_{(0)}\right|-\frac{1}{4}+\frac{3}{4}+\left|y_{(1)}\right|,
\end{aligned}
$$

which yields $\left|y_{(0)}\right|+\left|y_{(1)}\right| \leq \frac{1}{2}+\varepsilon<\frac{3}{4}$. Thus since $\|y\|=1$ there must exist a subset $A$ of a branch or a $\lambda$-segment such that $|A \cap\{(0),(1)\}|=1$ and $\sum_{t \in A}\left|y_{t}\right|=1$. Let $s \in A \cap\{(0),(1)\}$.

$$
\frac{5}{4}>\|x \pm y\|=\max _{ \pm}\left|x_{s} \pm y_{s}\right|+\sum_{\substack{t \in A \\ t \neq s}}\left|y_{t}\right|=\left|x_{s}\right|+\left|y_{s}\right|+1-\left|y_{s}\right|
$$

and we get the contradiction $\left|x_{s}\right|<\frac{1}{4}$.

Recall from [HLP15] that a Banach space $X$ is locally octahedral if for every $x \in S_{X}$ and $\varepsilon>0$, there exists $y \in S_{X}$ such that $\|x \pm y\| \geq 2-\varepsilon$.

It is known that every Banach space with the Daugavet property is octahedral. Even though the modified binary tree space have lots of Daugavet-points, as seen in Proposition 4.5, it is not even locally octahedral.

Proposition 4.11. $X_{\mathfrak{M}}$ is not locally octahedral.

Proof. Consider $x=\frac{1}{2}\left(e_{(0)}+e_{(1)}\right) \in S_{X_{\mathfrak{M}}}$. We want to show that for all $y \in S_{X_{\mathfrak{M}}}$ we have $\min \|x \pm y\| \leq \frac{3}{2}$. 
Let $y=\sum_{t \in \mathfrak{M}} y_{t} e_{t} \in S_{X_{\mathfrak{M}}}$. Let $A$ be a subset of a branch or a $\lambda$-segment. If $A \neq\{(0),(1)\}$, then

$$
\sum_{t \in A}\left|x_{t} \pm y_{t}\right| \leq\left\{\begin{array}{ll}
\frac{1}{2}+\sum_{t \in A}\left|y_{t}\right| ; & A \cap\{(0),(1)\} \neq \emptyset \\
\sum_{t \in A}\left|y_{t}\right| ; & A \cap\{(0),(1)\}=\emptyset
\end{array} \leq\left\{\begin{array}{l}
\frac{3}{2} \\
1
\end{array}\right.\right.
$$

If $A=\{(0),(1)\}$, then, since $\left|y_{(0)}\right|+\left|y_{(1)}\right| \leq 1$ and a convex function attains its maximum at the extreme points, we get

$$
\left|\frac{1}{2}+y_{(0)}\right|+\left|\frac{1}{2}+y_{(1)}\right|+\left|\frac{1}{2}-y_{(0)}\right|+\left|\frac{1}{2}-y_{(1)}\right| \leq 3
$$

Hence $\min \|x \pm y\| \leq \frac{3}{2}$.

\section{REFERENCES}

[AHLP20] T. A. Abrahamsen, R. Haller, V. Lima, and K. Pirk, Delta- and Daugavet points in Banach spaces, Proc. Edinb. Math. Soc. (2) 63 (2020), no. 2, 475-496, DOI 10.1017/s0013091519000567. MR4085036

[ALL16] Trond A. Abrahamsen, Johann Langemets, and Vegard Lima, Almost square Banach spaces, J. Math. Anal. Appl. 434 (2016), no. 2, 1549-1565, DOI 10.1016/j.jmaa.2015.09.060. MR3415738

[AM93] S. Argyros and S. Mercourakis, On weakly Lindelöf Banach spaces, Rocky Mountain J. Math. 23 (1993), no. 2, 395-446, DOI 10.1216/rmjm/1181072569. MR.1226181

[AT04] Spiros A. Argyros and Andreas Tolias, Methods in the theory of hereditarily indecomposable Banach spaces, Mem. Amer. Math. Soc. 170 (2004), no. 806, vi+114, DOI 10.1090/memo/0806. MR2053392

[BGLPRZ15] Julio Becerra Guerrero, Ginés López-Pérez, and Abraham Rueda Zoca, Big slices versus big relatively weakly open subsets in Banach spaces, J. Math. Anal. Appl. 428 (2015), no. 2, 855-865, DOI 10.1016/j.jmaa.2015.03.056. MR.3334951

[BGLPRZ18] Julio Becerra Guerrero, Ginés López-Pérez, and Abraham Rueda Zoca, Diametral diameter two properties in Banach spaces, J. Convex Anal. 25 (2018), no. 3, 817840, DOI 10.1017/s0308210517000373. MR3818544

[HLP15] Rainis Haller, Johann Langemets, and Märt Põldvere, On duality of diameter 2 properties, J. Convex Anal. 22 (2015), no. 2, 465-483. MR3346197

[HPV20] Rainis Haller, Katriin Pirk, and Triinu Veeorg, Daugavet- and delta-points in absolute sums of Banach spaces, J. Convex Anal. 28 (2021), no. 1, 41-54. MR 4199633

[IK04] Y. Ivakhno and V. Kadets, Unconditional sums of spaces with bad projections, Visn. Khark. Univ., Ser. Mat. Prykl. Mat. Mekh. 645 (2004), no. 54, 30-35 (English).

[Kad96] Vladimir M. Kadets, Some remarks concerning the Daugavet equation, Quaestiones Math. 19 (1996), no. 1-2, 225-235. MR1390483

[LT77] Joram Lindenstrauss and Lior Tzafriri, Classical Banach spaces. I: Sequence spaces, Ergebnisse der Mathematik und ihrer Grenzgebiete, Vol. 92, Springer-Verlag, BerlinNew York, 1977. MR0500056

[Shv00] R. V. Shvydkoy, Geometric aspects of the Daugavet property, J. Funct. Anal. 176 (2000), no. 2, 198-212, DOI 10.1006/jfan.2000.3626. MR.1784413

[Tal79] Michel Talagrand, Espaces de Banach faiblement $\mathcal{K}$-analytiques (French), Ann. of Math. (2) 110 (1979), no. 3, 407-438, DOI 10.2307/1971232. MR554378

[Tal84] Michel Talagrand, A new countably determined Banach space, Israel J. Math. 47 (1984), no. 1, 75-80, DOI 10.1007/BF02760563. MR736065

[Wer01] Dirk Werner, Recent progress on the Daugavet property, Irish Math. Soc. Bull. 46 (2001), 77-97. MR.1856978 
Department of Mathematics, University of Agder, Postboks 422, 4604 Kristiansand, NORWAY

Email address: trond.a.abrahamsen@uia.no

$U R L:$ http://home.uia.no/trondaa/index.php3

Department of Engineering Sciences, University of Agder, Postboks 509, 4898 GrimSTAD, NORWAY

Email address: Vegard.Lima@uia.no

Department of Mathematics, University of Agder, Postboks 422, 4604 Kristiansand, NoRWAY

Email address: andre.martiny@uia.no

Institute of Mathematics and Informatics, Bulgarian Academy of Science, Bl.8, ACAd. G. Bonchev str. 1113 Sofia, Bulgaria; and Departamento de Matemáticas, Universidad de Murcia, Campus de Espinardo, 30100 Espinardo (Murcia), Spain

Email address: stroya@um.es 